\title{
The Commutators of Fractional Integrals on Generalized Herz Spaces
}

\author{
Yue Hu, ${ }^{1}$ Yuexiang $\mathrm{He}^{2}{ }^{2}$ and Yueshan Wang ${ }^{2}$ \\ ${ }^{1}$ College of Mathematics and Informatics, Henan Polytechnic University, Jiaozuo 454003, China \\ ${ }^{2}$ Department of Mathematics, Jiaozuo University, Jiaozuo 454003, China
}

Correspondence should be addressed to Yue Hu; huu3y3@163.com

Received 7 February 2014; Accepted 14 March 2014; Published 3 April 2014

Academic Editor: Dragan Djordjevic

Copyright (c) 2014 Yue Hu et al. This is an open access article distributed under the Creative Commons Attribution License, which permits unrestricted use, distribution, and reproduction in any medium, provided the original work is properly cited.

Let $I_{l}$ be the fractional integral, $0<l<n$ and let $b \in \mathrm{BMO}$. We will obtain the weighted estimates for the commutator $\left[b, I_{l}\right]$ on the generalized Herz spaces.

\section{Introduction and Results}

Let $0<l<n$ the fractional integral operator $I_{l}$ is defined by

$$
I_{l} f(x)=\int_{\mathbb{R}^{n}} \frac{f(y)}{|x-y|^{n-l}} d y .
$$

Consider the commutator associated with the fractional integrals $I_{l}$ and the locally integrable function $b$,

$$
\left[b, I_{l}\right] f(x)=b(x) I_{l} f(x)-I_{l}(b f)(x) .
$$

The area of the commutators of fractional integrals has been under intensive research. When $b \in \mathrm{BMO}\left(\mathbb{R}^{n}\right), 1 / p_{2}=1 / p_{1}-$ $l / n$ and $1<p_{1}<n / l$, and Chanillo proved in [1] that $\left[b, I_{l}\right]$ is bounded from $L^{p_{1}}\left(\mathbb{R}^{n}\right)$ to $L^{p_{2}}\left(\mathbb{R}^{n}\right)$. Segovia and Torrea in [2] proved that $\left[b, I_{l}\right]$ is also bounded from $L^{p_{1}}\left(\omega^{p_{1}}, \mathbb{R}^{n}\right)$ to $L^{p_{2}}\left(\omega^{p_{2}}, \mathbb{R}^{n}\right)$ if $\omega \in A_{p_{1}, p_{2}}\left(\mathbb{R}^{n}\right)$. Lu and Yang in [3] studied $\left[b, I_{l}\right]$ Herz spaces; they proved that if $1 / p_{2}=1 / p_{1}-l / n, 1<$ $p_{1}<n / l,-n / p_{1}+l<\alpha<n\left(1-1 / p_{1}\right)$ and $0<q_{1} \leq q_{2}<\infty$, then $\left[b, I_{l}\right]$ maps $\dot{K}_{p_{1}}^{\alpha, q_{1}}\left(\mathbb{R}^{n}\right)$ continuously into $\dot{K}_{p_{2}}^{\alpha, q_{2}}\left(\mathbb{R}^{n}\right)$.

In [4], Komori and Matsuoka considered the boundedness of singular integral operators and fractional integral operators on weighted Herz spaces; they introduced generalized Herz spaces, and, as corollaries of their general theory, they obtained the boundedness of these operators on weighted Herz spaces.
For a sequence $\varphi=\{\varphi(k)\}_{-\infty}^{\infty}, \varphi(k)>0$, we suppose that $\varphi$ satisfies doubling condition of order $(s, t)$ and write $\varphi \in$ $D(s, t)$ if there exists $C \geq 1$ such that

$$
C^{-1} 2^{s(k-j)} \leq \frac{\varphi(k)}{\varphi(j)} \leq C 2^{t(k-j)},
$$

for $k>j$.

Let $B_{k}=\left\{x \in \mathbb{R}^{n}:|x| \leq 2^{k}\right\}, C_{k}=B_{k} \backslash B_{k-1}$ and let $\chi_{k}=$ $\chi_{C_{k}}$ be the characteristic function of the set $C_{k}$ for any $k \in \mathbb{Z}$. Suppose that $\omega$ is a weight function on $\mathbb{R}^{n}$. For $1<p<\infty$, $0<q<\infty$, the generalized Herz space is defined by

$$
\begin{gathered}
K_{p}^{q}(\varphi, \omega)\left(\mathbb{R}^{n}\right) \\
=\left\{f: f \text { is a measurable function on } \mathbb{R}^{n},\right. \\
\left.\|f\|_{K_{p}^{q}(\varphi, \omega)}<\infty\right\},
\end{gathered}
$$

where

$$
\begin{aligned}
\|f\|_{K_{p}^{q}(\varphi, \omega)} & =\left(\sum_{k=-\infty}^{+\infty} \varphi(k)^{q}\left\|f \chi_{k}\right\|_{L^{p}(\omega)}^{q}\right)^{1 / q}, \\
\|f\|_{L^{p}(\omega)} & =\left(\int_{\mathbb{R}^{n}}|f(x)|^{q} \omega(x) d x\right)^{1 / q} .
\end{aligned}
$$


Let $\varphi(k)=\omega_{1}\left(B_{k}\right)^{\alpha / n}, \omega=\omega_{2}$, and $\omega_{1}, \omega_{2}$ be weight functions in (5); then, $K_{p}^{q}(\varphi, \omega)\left(\mathbb{R}^{n}\right)$ is the weighted Herz spaces $\dot{K}_{p}^{\alpha}\left(\omega_{1}, \omega_{2}\right)\left(\mathbb{R}^{n}\right)$ (see [5]).

Let $\delta>0$. We say $\omega \in R D(\delta)$ (centered reverse doubling) if there is a positive number $C$ such that

$$
\frac{\omega\left(B_{k}\right)}{\omega\left(B_{j}\right)} \geq C 2^{\delta(k-j)} \quad \text { for } k>j .
$$

In 2000, Lu et al. in [6] proved boundedness results for sublinear operators on weighted Herz spaces with general Muckenhoupt weights. Recently, many authors considered the boundedness of operators on weighted Herz spaces. In [7], Wang proved the boundedness properties of intrinsic square function on weighted Herz spaces. Tomita in [8] studied the Strang-Fix theory for approximation order in weighted Herz spaces with general Muckenhoupt weights. In [9], $\mathrm{Hu}$ and Wang consider the boundedness properties of commutator operators generated by $\mathrm{BMO}\left(\mathbb{R}^{n}\right)$ function and intrinsic square function on weighted Herz spaces.

In this paper, we investigate the boundedness of commutators generated by fractional integrals and $\operatorname{BMO}\left(\mathbb{R}^{n}\right)$ on generalized Herz spaces $K_{p}^{q}(\varphi, \omega)\left(\mathbb{R}^{n}\right)$ with general Muckenhoupt weights. Our main results in this paper are formulated as follows.

Theorem 1. Let $b \in B M O\left(\mathbb{R}^{n}\right), 1<p_{1}<n / l, 0<q<\infty$, $\delta>0$ and let $1 / p_{2}=1 / p_{1}-l / n$. One assumes that

(1) $\varphi \in D(a, b)$, where $-\left(\delta / p_{2}\right)<a \leq b<n\left(1-\left(1 / p_{1}\right)\right)$;

(2) $\omega^{p_{2}} \in A_{r}$, where $r=\min \left(1+\left(p_{2} / p_{1}^{\prime}\right), 1+\left(p_{2} / p_{1}^{\prime}\right)-\right.$ $\left.\left(b p_{2} / n\right)\right)$;

(3) $\omega^{p_{2}} \in R D(\delta)$.

Then, $\left[b, I_{l}\right]$ is bounded from $K_{p_{1}}^{q}\left(\varphi, \omega^{p_{1}}\right)\left(\mathbb{R}^{n}\right)$ to $K_{p_{2}}^{q}(\varphi$, $\left.\omega^{p_{2}}\right)\left(\mathbb{R}^{n}\right)$.

Remark 2. When $b \leq 0$, condition (2) is equivalent to the condition that $\omega \in A_{p_{1}, p_{2}}\left(\mathbb{R}^{n}\right)$, but when $b>0$, (2) is stronger than $A_{p_{1}, p_{2}}$-condition. Komori and Matsuoka in [4] showed that the condition (2) is the best possible by a counterexample.

Let $\varphi \in D(0, \alpha)$ in Theorem 1; we have

Corollary 3. Let $1<p_{1}<n / l, 0<q<\infty, 0 \leq \alpha<$ $n\left(1-1 / p_{1}\right)$ and let $1 / p_{2}=1 / p_{1}-l / n$. If $b \in B M O\left(\mathbb{R}^{n}\right)$, $\omega^{p_{2}} \in A_{1+p_{2} / p_{1}^{\prime}-\alpha p_{2} / n}\left(\mathbb{R}^{n}\right)$, then $\left[b, I_{l}\right]$ is bounded from $\dot{K}_{p_{1}}^{\alpha, q}\left(1, \omega^{p_{1}}\right)\left(\mathbb{R}^{n}\right)$ to $\dot{K}_{p_{2}}^{\alpha, q}\left(1, \omega^{p_{2}}\right)\left(\mathbb{R}^{n}\right)$.

The paper is organized as follows. In Section 2, we give some basic notation and definitions and recall some preliminaries. In Section 3, we will prove Theorem 1.

\section{Definitions and Preliminaries}

We begin with some properties of $A_{p}$ weights which play a great role in the proofs of our main results.

A weight $\omega$ is a nonnegative, locally integrable function on $\mathbb{R}^{n}$. Let $B=B\left(x_{0}, r_{B}\right)$ denote the ball with the center $x_{0}$ and radius $r_{B}$. For any ball $B$ and $\lambda>0, \lambda B$ denotes the ball concentric with $B$ whose radius is $\lambda$ times as long. For a given weight function $\omega$ and a measurable set $E$, we also denote the Lebesgue measure of $E$ by $|E|$ and set the weighted measure $\omega(E)=\int_{E} \omega(x) d x$.

A weight $\omega$ is said to belong to $A_{p}\left(\mathbb{R}^{n}\right)$ for $1<p<\infty$, if there exists a constant $C$ such that for every ball $B \subset \mathbb{R}^{n}$,

$$
\left(\frac{1}{|B|} \int_{B} \omega(x) d x\right)\left(\frac{1}{|B|} \int_{B} \omega(x)^{1-p^{\prime}} d x\right)^{p-1} \leq C,
$$

where $s^{\prime}$ is the dual of $s$ such that $1 / s+1 / s^{\prime}=1$. The class $A_{1}\left(\mathbb{R}^{n}\right)$ is defined by replacing the above inequality with

$$
\frac{1}{|B|} \int_{B} w(y) d y \leq C \cdot \underset{x \in B}{\operatorname{ess} \inf } w(x)
$$

$$
\text { for every ball } B \subset \mathbb{R}^{n} \text {. }
$$

A weight $\omega$ is said to belong to $A_{\infty}\left(\mathbb{R}^{n}\right)$ if there are positive numbers $C$ and $\delta$ so that

$$
\frac{\omega(E)}{\omega(B)} \leq C\left(\frac{|E|}{|B|}\right)^{\delta}
$$

for all balls $B$ and all measurable $E \subset B$. It is well known that

$$
A_{\infty}\left(\mathbb{R}^{n}\right)=\bigcup_{1 \leq p<\infty} A_{p}\left(\mathbb{R}^{n}\right) .
$$

The classical $A_{p}\left(\mathbb{R}^{n}\right)$ weight theory was first introduced by Muckenhoupt in the study of weighted $L^{p}$ boundedness of Hardy-Littlewood maximal function in [10].

Lemma 4 (see $[10,12])$. Suppose $\omega \in A_{p}\left(\mathbb{R}^{n}\right)$ and the following statements hold.

(i) For any $1 \leq p<\infty$, there are positive numbers $C$ and $\delta$ such that

$$
\begin{array}{ll}
\frac{\omega\left(B_{k}\right)}{\omega\left(B_{j}\right)} \leq C 2^{n p(k-j)} & \text { for } k>j, \\
\frac{\omega\left(B_{k}\right)}{\omega\left(B_{j}\right)} \leq C 2^{n p \delta(k-j)} & \text { for } k<j ;
\end{array}
$$

(ii) $A_{p_{1}}\left(\mathbb{R}^{n}\right) \subset A_{p_{2}}\left(\mathbb{R}^{n}\right)$ for any $1 \leq p_{1}<p_{2} \leq \infty$;

(iii) For any $1<p<\infty$, one has $\omega^{1-p^{\prime}} \in A_{p^{\prime}}\left(\mathbb{R}^{n}\right)$.

We also need another weight class $A_{p, q}\left(\mathbb{R}^{n}\right)$ introduced by Muckenhoupt and Wheeden in [11] to study weighted boundedness of fractional integral operators.

Given $1 \leq p \leq q<\infty$, we say that $\omega \in A_{p, q}\left(\mathbb{R}^{n}\right)$ if there exists a constant $C$ such that for every ball $B \subset \mathbb{R}^{n}$, the inequality

$$
\left(\frac{1}{|B|} \int_{B} \omega(y)^{-p^{\prime}} d y\right)^{1 / p^{\prime}}\left(\frac{1}{|B|} \int_{B} \omega(y)^{q} d y\right)^{1 / q} \leq C
$$


holds when $1<p<\infty$, and the inequality

$$
\begin{array}{r}
\left(\frac{1}{|B|} \int_{B} \omega(y)^{q} d y\right)^{1 / q} \leq C \cdot \underset{x \in B}{\text { ess inf }} w(x) \\
\text { for every ball } B \subset \mathbb{R}^{n}
\end{array}
$$

holds when $p=1$.

By (13), we have

$$
\left(\int_{B} \omega(y)^{-p^{\prime}} d y\right)^{1 / p^{\prime}}\left(\int_{B} \omega(y)^{q} d y\right)^{1 / q} \leq C|B|^{1 / p^{\prime}+1 / q} .
$$

We summarize some properties about weights $A_{p, q}\left(\mathbb{R}^{n}\right)$ (see $[11,12])$.

Lemma 5. Given $1 \leq p \leq q<\infty$,

(i) $\omega \in A_{p, q}\left(\mathbb{R}^{n}\right)$ if and only if $\omega^{q} \in A_{1+q / p^{\prime}}\left(\mathbb{R}^{n}\right)$;

(ii) $\omega \in A_{p, q}\left(\mathbb{R}^{n}\right)$ if and only if $\omega^{-p^{\prime}} \in A_{1+p^{\prime} / q}\left(\mathbb{R}^{n}\right)$;

(iii) $\omega \in A_{p, p}\left(\mathbb{R}^{n}\right)$ if and only if $\omega^{p} \in A_{p}\left(\mathbb{R}^{n}\right)$;

(iv) If $p_{1}<p_{2}$ and $q_{2}>q_{1}$, then $A_{p_{1}, q_{1}}\left(\mathbb{R}^{n}\right) \subset A_{p_{2}, q_{2}}\left(\mathbb{R}^{n}\right)$. if

A locally integrable function $b$ is said to be in $\operatorname{BMO}\left(\mathbb{R}^{n}\right)$

$$
\sup _{B \subset \mathbb{R}^{n}} \frac{1}{|B|} \int_{B}\left|b(x)-b_{B}\right| d x=\|b\|_{*}<\infty,
$$

where $b_{B}=|B|^{-1} \int_{B} b(y) d y$.

Lemma 6 (John-Nirenberg inequality; see [13]). Let $b \in$ $B M O\left(\mathbb{R}^{n}\right)$. Then, for any ball $B \subset \mathbb{R}^{n}$, there exist positive constants $C_{1}$ and $C_{2}$ such that, for all $\lambda>0$,

$$
\left|\left\{x \in B:\left|b(x)-b_{B}\right|>\lambda\right\}\right| \leq C_{1}|B| \exp \left(-\frac{C_{2} \lambda}{\|b\|_{*}}\right) .
$$

Lemma 7 (see [14]). Let $\omega \in A_{\infty}\left(\mathbb{R}^{n}\right)$. Then, the norm of $B M O\left(\omega, \mathbb{R}^{n}\right)$ is equivalent to the norm of $B M O\left(\mathbb{R}^{n}\right)$, where

$$
\begin{gathered}
\operatorname{BMO}\left(\omega, \mathbb{R}^{n}\right) \\
=\left\{b:\|b\|_{*, \omega}=\sup _{B \subset \mathbb{R}^{n}} \frac{1}{\omega(B)}\right. \\
\left.\times \int_{B}\left|b(x)-b_{B, \omega}\right| \omega(x) d x<\infty\right\}, \\
b_{B, \omega}=\frac{1}{\omega(B)} \int_{B} b(z) \omega(z) d z .
\end{gathered}
$$

Lemma 8. Suppose $\omega \in A_{\infty}\left(\mathbb{R}^{n}\right)$ and $b \in B M O\left(\mathbb{R}^{n}\right)$. Then, for any $p \geq 1$, one has

$$
\left(\frac{1}{\omega(B)} \int_{B}\left|b(x)-b_{B, \omega}\right|^{p} \omega(x) d x\right)^{1 / p} \leq C\|b\|_{*} .
$$

Proof. Since $\omega(x) \in A_{\infty}\left(\mathbb{R}^{n}\right),(10)$ and Lemma 6 imply that

$$
\omega\left(\left\{x \in B:\left|b(x)-b_{B}\right|>\lambda\right\}\right) \leq C \omega(B) \exp \left(-\frac{C_{2} \delta \lambda}{\|b\|_{*}}\right) .
$$

Therefore,

$$
\begin{aligned}
& \int_{B}\left|b(x)-b_{B}\right|^{p} \omega(x) d x \\
& \leq p \int_{0}^{\infty} \lambda^{p-1} \omega\left(\left\{x \in B:\left|b(x)-b_{B}\right|>\lambda\right\}\right) d \lambda \\
& \leq C \omega(B) \int_{0}^{\infty} \lambda^{p-1} \exp \left(-\frac{C_{2} \delta \lambda}{\|b\|_{*}}\right) d \lambda \\
& \leq C \omega(B)\|b\|_{*}^{p} .
\end{aligned}
$$

Hence,

$$
\begin{aligned}
& \frac{1}{\omega(B)} \int_{B}\left|b(x)-b_{B, \omega}\right|^{p} \omega(x) d x \\
& \leq \frac{C}{\omega(B)} \int_{B}\left|b(x)-b_{B}\right|^{p} \omega(x) d x \\
& \leq C\|b\|_{*}^{p} .
\end{aligned}
$$

\section{Proof of Theorem 1}

Let $f \in K_{p_{1}}^{q}\left(\varphi, \omega^{p_{1}}\right)\left(\mathbb{R}^{n}\right), b \in \operatorname{BMO}\left(\mathbb{R}^{n}\right)$. Then,

$$
\begin{aligned}
& \left\|\left[b, I_{l}\right] f\right\|_{K_{p_{2}}^{q}\left(\varphi, \omega^{p_{2}}\right)}^{q} \\
& \leq C \sum_{j=-\infty}^{\infty} \varphi(j)^{q}\left(\sum_{k=-\infty}^{j-2}\left\|\left[b, I_{l}\right]\left(f \chi_{k}\right) \chi_{j}(x)\right\|_{L^{p_{2}}\left(\omega^{p_{2}}\right)}\right)^{q} \\
& +C \sum_{j=-\infty}^{\infty} \varphi(j)^{q}\left(\sum_{k=j-1}^{j+1}\left\|\left[b, I_{l}\right]\left(f \chi_{k}\right) \chi_{j}(x)\right\|_{L^{p_{2}}\left(\omega^{p_{2}}\right)}\right)^{q} \\
& +C \sum_{j=-\infty}^{\infty} \varphi(j)^{q}\left(\sum_{k=j+2}^{\infty}\left\|\left[b, I_{l}\right]\left(f \chi_{k}\right) \chi_{j}(x)\right\|_{L^{p_{2}}\left(\omega^{p_{2}}\right)}\right)^{q} \\
& =L_{1}+L_{2}+L_{3} .
\end{aligned}
$$

Since $\omega^{p_{2}} \in A_{r}$, where $r=\min \left\{1+\left(p_{2} / p_{1}^{\prime}\right), 1+\right.$ $\left.\left(p_{2} / p_{1}^{\prime}\right)-\left(b p_{2} / n\right)\right\}$, then, by (i) of Lemma 5 , we know that $\omega \in$ $A_{p_{1}, p_{2}}\left(\mathbb{R}^{n}\right)$. Using the fact that $\left[b, I_{l}\right]$ is a bounded operator from $L^{p_{1}}\left(\omega^{p_{1}}, \mathbb{R}^{n}\right)$ to $L^{p_{2}}\left(\omega^{p_{2}}, \mathbb{R}^{n}\right)$ (see [2]), we obtain

$$
\begin{aligned}
L_{2} & =C \sum_{j=-\infty}^{\infty} \varphi(j)^{q}\left(\sum_{k=j-1}^{j+1}\left\|\left[b, I_{l}\right]\left(f \chi_{k}\right) \chi_{j}(x)\right\|_{L^{p_{2}}\left(\omega^{p_{2}}\right)}\right)^{q} \\
& \leq C\|b\|_{*}^{q} \sum_{j=-\infty}^{\infty} \varphi(j)^{q} \sum_{k=j-1}^{j+1}\left\|f \chi_{k}\right\|_{L^{p_{1}}\left(\omega^{p_{1}}\right)}^{q}
\end{aligned}
$$$$
\leq C\|b\|_{*}^{q} \sum_{j=-\infty}^{\infty} \varphi(j)^{q}\left\|f \chi_{j}\right\|_{L^{p_{1}\left(\omega^{p_{1}}\right)}}^{q}
$$$$
\leq C\|b\|_{*}^{q}\|f\|_{K_{p_{1}}^{q}\left(\varphi, \omega^{p_{1}}\right)}^{q} .
$$ 
We now estimate $L_{1}$. Obviously,

$$
\begin{aligned}
& \left\|\left[b, I_{l}\right]\left(f \chi_{k}\right) \chi_{j}(x)\right\|_{L^{p_{2}}\left(\omega^{p_{2}}\right)} \\
& \leq C\left(\int_{C_{j}} \mid\left(b(x)-b_{B_{k}}\right) \int_{C_{k}} \frac{1}{|x-y|^{n-l}}\right. \\
& \left.\quad \times\left(f \chi_{k}\right)(y) d y||^{p_{2}} \omega(x)^{p_{2}} d x\right)^{1 / p_{2}} \\
& +C\left(\int_{C_{j}} \mid \int_{C_{k}} \frac{1}{|x-y|^{n-l}}\left(b(y)-b_{B_{k}}\right)\right. \\
& \left.\times\left.\left(f \chi_{k}\right)(y) d y\right|^{p_{2}} \omega(x)^{p_{2}} d x\right)^{1 / p_{2}} \\
& =M_{1}+M_{2} .
\end{aligned}
$$

First, we estimate $M_{1}$. From $j \geq k+2$, one can see then that

$$
\begin{aligned}
M_{1}=\left(\int_{C_{j}} \mid\left(b(x)-b_{B_{k}}\right) \int_{C_{k}} \frac{1}{|x-y|^{n-l}}\right. \\
\left.\quad \times\left.\left(f \chi_{k}\right)(y) d y\right|^{p_{2}} \omega(x)^{p_{2}} d x\right)^{1 / p_{2}} \\
\leq C 2^{-j(n-l)}\left\|f \chi_{k}\right\|_{L^{1}} \\
\times\left(\left(\int_{B_{j}}\left|b(x)-b_{B_{j}, \omega^{p_{2}}}\right|^{p_{2}} \omega(x)^{p_{2}} d x\right)^{1 / p_{2}}\right. \\
\quad+\left(\left|b_{B_{j}}-b_{B_{j}, \omega^{p_{2}}}\right|+\left|b_{B_{j}}-b_{B_{k}}\right|\right) \\
\left.\times\left(\int_{B_{j}} \omega(x)^{p_{2}} d x\right)^{1 / p_{2}}\right) .
\end{aligned}
$$

Since $\omega^{p_{2}} \in A_{r}$, then, by Lemma 8 ,

$$
\begin{gathered}
\left(\int_{B_{j}}\left|b(x)-b_{B_{j}, \omega^{p_{2}}}\right|^{p_{2}} \omega(x)^{p_{2}} d x\right)^{1 / p_{2}} \\
\leq C\|b\|_{*}\left(\int_{B_{j}} \omega(x)^{p_{2}} d x\right)^{1 / p_{2}} .
\end{gathered}
$$

Similar to the proof of Lemma 8, we can deduce that

$$
\left|b_{B_{j}}-b_{B_{j}, \omega^{p 2}}\right| \leq C\|b\|_{*} \text {. }
$$

From the definition of $\mathrm{BMO}\left(\mathbb{R}^{n}\right)$, it is easy to see that

$$
\left|b_{B_{j}}-b_{B_{k}}\right| \leq C(j-k)\|b\|_{*} \text {. }
$$

By Hölder's inequality, we get

$$
\left\|f \chi_{k}\right\|_{L^{1}} \leq C\left\|f \chi_{k}\right\|_{L^{p_{1}}\left(\omega^{p_{1}}\right)}\left(\int_{B_{k}} \omega^{-p_{1}^{\prime}} d x\right)^{1 / p_{1}^{\prime}} .
$$

Then,

$$
\begin{aligned}
M_{1} \leq C & (j-k) 2^{-j(n-l)}\|b\|_{*}\left\|f \chi_{k}\right\|_{L^{p_{1}}\left(\omega^{p_{1}}\right)} \\
& \times\left(\int_{B_{k}} \omega^{-p_{1}^{\prime}} d x\right)^{1 / p_{1}^{\prime}}\left(\int_{B_{j}} \omega(x)^{p_{2}} d x\right)^{1 / p_{2}} .
\end{aligned}
$$

We now turn to estimate $M_{2}$. Since $\omega \in A_{p_{1}, p_{2}}\left(\mathbb{R}^{n}\right)$, then, by (ii) of Lemma 5 , we know $\omega^{-p_{1}^{\prime}} \in A_{\infty}\left(\mathbb{R}^{n}\right)$. Similar to the proof of Lemma 8, we have

$$
\begin{gathered}
\left(\int_{B_{k}}\left|b(x)-b_{B_{k}}\right|^{p_{1}^{\prime}} \omega^{-p_{1}^{\prime}} d x\right)^{1 / p_{2}} \\
\leq C\|b\|_{*}\left(\int_{B_{k}} \omega(x)^{-p_{1}^{\prime}} d x\right)^{1 / p_{1}^{\prime}} .
\end{gathered}
$$

Using Hölder's inequality and (32),

$$
\begin{aligned}
M_{2} \leq & C 2^{-j(n-l)} \int_{B_{k}}\left|b(y)-b_{B_{k}}\right|\left|f \chi_{k}(y)\right| d y \\
& \times\left(\int_{B_{j}} \omega(x)^{p_{2}} d x\right)^{1 / p_{2}}
\end{aligned}
$$

$$
\begin{aligned}
\leq & C 2^{-j(n-l)}\left(\int_{B_{k}}\left|b(y)-b_{B_{k}}\right|^{p_{1}^{\prime}} \omega(y)^{-p_{1}^{\prime}} d y\right)^{1 / p_{1}^{\prime}} \\
& \times\left\|f \chi_{k}\right\|_{L^{p_{1}\left(\omega^{\left.p_{1}\right)}\right.}}\left(\int_{B_{j}} \omega(x)^{p_{2}} d x\right)^{1 / p_{2}} \\
\leq & C 2^{-j(n-l)}\|b\|_{*}\left\|f \chi_{k}\right\|_{L^{p_{1}}\left(\omega^{p_{1}}\right)} \\
& \times\left(\int_{B_{k}} \omega(x)^{-p_{1}^{\prime}} d x\right)^{1 / p_{1}^{\prime}}\left(\int_{B_{j}} \omega(x)^{p_{2}} d x\right)^{1 / p_{2}} .
\end{aligned}
$$

Since $\omega(x)^{p_{2}} \in A_{r}$, by (iii) of Lemma 4 , we have $\omega(x)^{p_{2}} \in A_{\bar{r}}$ for some $\bar{r}<r$. Then, from (15) and (i) of Lemma 4,

$$
\begin{aligned}
& \left(\int_{B_{k}} \omega(x)^{-p_{1}^{\prime}} d x\right)^{1 / p_{1}^{\prime}}\left(\int_{B_{j}} \omega^{p_{2}} d x\right)^{1 / p_{2}} \\
& =\left(\int_{B_{k}} \omega^{-p_{1}^{\prime}} d x\right)^{1 / p_{1}^{\prime}}\left(\int_{B_{k}} \omega(x)^{p_{2}} d x\right)^{1 / p_{2}} \\
& \quad \times\left(\frac{\omega^{p_{2}}\left(B_{j}\right)}{\omega^{p_{2}}\left(B_{k}\right)}\right)^{1 / p_{2}} \\
& \leq C 2^{k n\left(1 / p_{1}^{\prime}+1 / p_{2}\right)+(j-k) n \bar{r} / p_{2}} .
\end{aligned}
$$

Hence,

$$
\begin{aligned}
&\left\|\left[b, I_{l}\right]\left(f \chi_{k}\right) \chi_{j}(x)\right\|_{L^{p_{2}}\left(\omega^{p_{2}}\right)} \\
& \leq C\|b\|_{*}\left\|f \chi_{k}\right\|_{L^{p_{1}\left(\omega^{p_{1}}\right)}}(j-k) 2^{-j(n-l)} \\
& \quad \times\left(\int_{B_{k}} \omega^{-p_{1}^{\prime}} d x\right)^{1 / p_{1}^{\prime}}\left(\int_{B_{j}} \omega(x)^{p_{2}} d x\right)^{1 / p_{2}} \\
& \leq C\|b\|_{*}\left\|f \chi_{k}\right\|_{L^{p_{1}}\left(\omega^{p_{1}}\right)}(j-k) 2^{-j(n-l)} \\
& \leq C\|b\|_{*}\left\|f \chi_{k}\right\|_{L^{p_{1}}\left(\omega^{p_{1}}\right)} \\
& \times(j-k) 2^{j\left(-n+l+n \bar{r} / p_{2}\right)} 2^{n k\left(1 / p_{1}^{\prime}+1 / p_{2}-\bar{r} / p_{2}\right)} \\
&= C\|b\|_{*}\left\|f \chi_{k}\right\|_{L^{p_{1}\left(\omega^{p_{1}}\right)}}(j-k) 2^{(j-k)\left(-n+l+n \bar{r} / p_{2}\right)} .
\end{aligned}
$$


Using the condition that $\varphi \in D(a, b)$, we obtain

$$
\begin{aligned}
& L_{1}= C \sum_{j=-\infty}^{\infty} \varphi(j)^{q}\left(\sum_{k=-\infty}^{j-2}\left\|\left[b, I_{l}\right]\left(f \chi_{k}\right) \chi_{j}(x)\right\|_{L^{p_{2}}\left(\omega^{p_{2}}\right)}\right)^{q} \\
& \leq C\|b\|_{*}^{q} \sum_{j=-\infty}^{\infty} \varphi(j)^{q} \\
& \times\left(\sum_{k=-\infty}^{j-2}\left\|f \chi_{k}\right\|_{L^{p_{1}}\left(\omega^{\left.p_{1}\right)}\right.}(j-k) 2^{(j-k)\left(-n+l+n \bar{r} / p_{2}\right)}\right)^{q} \\
& \leq C\|b\|_{*}^{q} \sum_{j=-\infty}^{\infty}\left(\sum_{k=-\infty}^{j-2} \varphi(k)\left\|f \chi_{k}\right\|_{L^{p_{1}\left(\omega^{p_{1}}\right)}}\right. \\
&\left.\times(j-k) 2^{(j-k)\left(-n+l+n \bar{r} / p_{2}+b\right)}\right)^{q} .
\end{aligned}
$$

Since $r \leq 1+p_{2} / p_{1}^{\prime}-b p_{2} / n$, we have $-n+l+n r / p_{2}+b \leq 0$, and, then, $-n+l+n \bar{r} / p_{2}+b<0$. When $0<q \leq 1$, we have

$$
\begin{aligned}
& L_{1} \leq C\|b\|_{*}^{q} \sum_{k=-\infty}^{\infty} \varphi(k)^{q}\left\|f \chi_{k}\right\|_{L^{p_{1}}\left(\omega^{p_{1}}\right)}^{q} \\
& \times \sum_{j=k+2}^{\infty}(j-k)^{q} 2^{(j-k) q\left(-n+l+n \bar{r} / p_{2}+b\right)} \\
& \leq C\|b\|_{*}^{q} \sum_{k=-\infty}^{\infty} \varphi(k)^{q}\left\|f \chi_{k}\right\|_{L^{p_{1}}\left(\omega^{p_{1}}\right)}^{q} \\
& \leq C\|b\|_{*}^{q}\|f\|_{K_{p}^{q}\left(\mathbb{R}^{n}\right)^{q}}^{q}
\end{aligned}
$$

When $q>1$, by Hölder's inequality, we have

$$
\begin{aligned}
& L_{1} \leq C\|b\|_{*}^{q} \sum_{j=-\infty}^{\infty}\left(\sum_{k=-\infty}^{j-2} \varphi(k)^{q}\left\|f \chi_{k}\right\|_{L^{p_{1}\left(\omega^{p_{1}}\right)}}^{q}\right.\left.\times(j-k) 2^{(j-k)\left(-n+l+n \bar{r} / p_{2}+b\right)}\right) \\
& \times\left(\sum_{k=-\infty}^{j-2}(j-k) 2^{(j-k)\left(-n+l+n \bar{r} / p_{2}+b\right)}\right)^{q / q^{\prime}} \\
& \leq C\|b\|_{*}^{q} \sum_{k=-\infty}^{\infty} \varphi(k)^{q}\left\|f \chi_{k}\right\|_{L^{p_{1}}\left(\omega^{\left.p_{1}\right)}\right.}^{q} \\
& \leq C\|b\|_{*}^{q}\|f\|_{K_{p}^{q}\left(\mathbb{R}^{n}\right)^{.}}^{q}
\end{aligned}
$$

Now, we estimate $L_{3}$. Since $k \geq j+2$, similar to the estimation of $L_{1}$, we have

$$
\begin{aligned}
& \|\left[b, I_{l}\right]\left(f \chi_{k}\right) \chi_{j}(x) \|_{L^{p_{2}}\left(\omega^{p_{2}}\right)} \\
& \leq C(k-j) 2^{-k(n-l)}\|b\|_{*}\left\|f \chi_{k}\right\|_{L^{p_{1}}\left(\omega^{p_{1}}\right)} \\
& \quad \times\left(\int_{B_{k}} \omega(x)^{-p_{1}^{\prime}} d x\right)^{1 / p_{1}^{\prime}}\left(\int_{B_{j}} \omega(x)^{p_{2}} d x\right)^{1 / p_{2}} .
\end{aligned}
$$

From the fact $\omega \in A_{p_{1}, p_{2}}\left(\mathbb{R}^{n}\right)$ and (15), we get

$$
\begin{aligned}
& \left(\int_{B_{k}} \omega^{-p_{1}^{\prime}} d x\right)^{1 / p_{1}^{\prime}}\left(\int_{B_{k}} \omega(x)^{p_{2}} d x\right)^{1 / p_{2}} \\
& \leq C 2^{k n\left(1 / p_{1}^{\prime}+1 / p_{2}\right)} .
\end{aligned}
$$

By $\omega^{p_{2}} \in R D(\delta)$, we have

$$
\left(\frac{\omega^{p_{2}}\left(B_{j}\right)}{\omega^{p_{2}}\left(B_{k}\right)}\right)^{1 / p_{2}} \leq C 2^{(j-k) \delta / p_{2}} .
$$

Then,

$$
\begin{aligned}
& \left(\int_{B_{k}} \omega(x)^{-p_{1}^{\prime}} d x\right)^{1 / p_{1}^{\prime}}\left(\int_{B_{j}} \omega(x)^{p_{2}} d x\right)^{1 / p_{2}} \\
& =\left(\int_{B_{k}} \omega^{-p_{1}^{\prime}} d x\right)^{1 / p_{1}^{\prime}}\left(\int_{B_{k}} \omega(x)^{p_{2}} d x\right)^{1 / p_{2}} \\
& \quad \times\left(\frac{\omega^{p_{2}}\left(B_{j}\right)}{\omega^{p_{2}}\left(B_{k}\right)}\right)^{1 / p_{2}} \\
& \leq C 2^{k n\left(1 / p_{1}^{\prime}+1 / p_{2}\right)+(j-k) \delta / p_{2}} .
\end{aligned}
$$

Hence,

$$
\begin{aligned}
& \left\|\left[b, I_{l}\right]\left(f \chi_{k}\right) \chi_{j}(x)\right\|_{L^{p_{2}}\left(\omega^{p_{2}}\right)} \\
& \quad \leq C\|b\|_{*}(k-j) 2^{k n\left(1 / p_{1}^{\prime}+1 / p_{2}\right)+(j-k) \delta / p_{2}-k(n-l)} \\
& \quad=C\|b\|_{*}(k-j) 2^{(j-k) \delta / p_{2}}\left\|f \chi_{k}\right\|_{L^{p_{1}}\left(\omega^{p_{1}}\right)} .
\end{aligned}
$$

Using the condition that $\varphi \in D(a, b)$,

$$
\begin{gathered}
L_{3}=C \sum_{j=-\infty}^{\infty} \varphi(j)^{q}\left(\sum_{k=j+2}^{\infty} \|\left(\left[b, I_{l}\right]\left(f \chi_{k}\right) \chi_{j}(x) \|_{L^{p_{2}}\left(\omega^{p_{2}}\right)}\right)^{q}\right. \\
\leq C\|b\|_{*}^{q} \sum_{j=-\infty}^{\infty}\left(\sum_{k=j+2}^{\infty} \varphi(k)\left\|f \chi_{k}\right\|_{L^{p_{1}}\left(\omega^{p_{1}}\right)}\right. \\
\left.\times(k-j) 2^{(j-k)\left(\delta / p_{2}+a\right)}\right)^{q} .
\end{gathered}
$$

When $0<q \leq 1$, we have

$$
\begin{aligned}
& L_{3} \leq C\|b\|_{*}^{q} \sum_{k=-\infty}^{\infty} \varphi(k)^{q}\left\|f \chi_{k}\right\|_{L^{p_{1}}\left(\omega^{p_{1}}\right)}^{q} \\
& \times \sum_{j=-\infty}^{k-2}(k-j)^{q} 2^{(j-k)\left(\delta / p_{2}+a\right) q} \\
& \leq C\|b\|_{*}^{q} \sum_{k=-\infty}^{\infty} \varphi(k)^{q}\left\|f \chi_{k}\right\|_{L^{p_{1}}\left(\omega^{p_{1}}\right)}^{q} \\
& \leq C\|b\|_{*}\|f\|_{K_{p}^{q}\left(\varphi, \omega^{p_{1}}\right)}^{q}
\end{aligned}
$$


When $q>1$, by the Hölder inequality, we get

$$
\begin{aligned}
& L_{3} \leq C\|b\|_{*}^{q} \sum_{j=-\infty}^{\infty}\left(\sum_{k=j+2}^{\infty} \varphi(k)\left\|f \chi_{k}\right\|_{L^{p_{1}}\left(\omega^{p_{1}}\right)}\right. \\
& \left.\times(k-j) 2^{(j-k)\left(\delta / p_{2}+a\right)}\right)^{q} . \\
& \leq C\|b\|_{*}^{q} \sum_{j=-\infty}^{\infty}\left(\sum_{k=j+2}^{\infty} \varphi(k)^{q}\left\|f \chi_{k}\right\|_{L^{p_{1}}\left(\omega^{p_{1}}\right)}^{q}\right. \\
& \left.\times(k-j) 2^{(j-k)\left(\delta / p_{2}+a\right)}\right) \\
& \times\left(\sum_{k=j+2}^{\infty}(k-j) 2^{(j-k)\left(\delta / p_{2}+a\right)}\right)^{q / q^{\prime}} \\
& \leq C\|b\|_{*}^{q} \sum_{k=-\infty}^{\infty} \varphi(k)^{q}\left\|f \chi_{k}\right\|_{L^{p_{1}}\left(\omega^{p_{1}}\right)}^{q} \\
& \times\left(\sum_{j=-\infty}^{k-2}(k-j)^{q} 2^{(j-k)\left(\delta / p_{2}+a\right) q}\right) \\
& \leq C\|b\|_{*}^{q} \sum_{k=-\infty}^{\infty} \varphi(k)^{q}\left\|f \chi_{k}\right\|_{L^{p_{1}}\left(\omega^{p_{1}}\right)}^{q} \\
& \leq C\|b\|_{*}^{q}\|f\|_{K_{p}^{q}\left(\varphi, \omega^{p_{1}}\right)}^{q} .
\end{aligned}
$$

Combining the above estimates for $L_{1}, L_{2}$, and $L_{3}$, the proof of Theorem 1 is completed.

\section{Conflict of Interests}

The authors declare that they have no conflict of interests.

\section{Acknowledgment}

The authors wish to thank the referee for his/her useful suggestions for the improvement of the paper.

\section{References}

[1] S. Chanillo, "A note on commutators," Indiana University Mathematics Journal, vol. 31, no. 1, pp. 7-16, 1982.

[2] C. Segovia and J. L. Torrea, "Weighted inequalities for commutators of fractional and singular integrals," Publicacions Matemàtiques, vol. 35, no. 1, pp. 209-235, 1991.

[3] S. Lu and D. Yang, "The continuity of commutators on Herztype spaces," Michigan Mathematical Journal, vol. 44, no. 2, pp. 255-281, 1997.

[4] Y. Komori and K. Matsuoka, "Boundedness of several operators on weighted Herz spaces," Journal of Function Spaces and Applications, vol. 7, no. 1, pp. 1-12, 2009.

[5] S. Z. Lu and D. C. Yang, "The decomposition of weighted Herz space on $\mathbb{R}^{n}$ and its applications," Science in China A: Mathematics, Physics, Astronomy, vol. 38, no. 2, pp. 147-158, 1995.
[6] S. Lu, K. Yabuta, and D. Yang, "Boundedness of some sublinear operators in weighted Herz-type spaces," Kodai Mathematical Journal, vol. 23, no. 3, pp. 391-410, 2000.

[7] H. Wang, "The boundedness of intrinsic square functions on the weighted Herz spaces," Journal of Function Spaces, vol. 2014, Article ID 274521, 14 pages, 2014.

[8] N. Tomita, "Strang-fix theory for approximation order in weighted $L^{p}$-spaces and Herz spaces," Journal of Function Spaces and Applications, vol. 4, no. 1, pp. 7-24, 2006.

[9] Y. Hu and Y. Wang, "The commutators of intrinsic square functions on weighted Herz spaces," The Bulletin of the Malaysian Mathematical Society 2. In press.

[10] B. Muckenhoupt, "Weighted norm inequalities for the Hardy maximal function," Transactions of the American Mathematical Society, vol. 165, pp. 207-226, 1972.

[11] B. Muckenhoupt and R. Wheeden, "Weighted norm inequalities for fractional integrals," Transactions of the American Mathematical Society, vol. 192, pp. 261-274, 1974.

[12] J. García-Cuerva and J. L. R. de Francia, Weighted Norm Inequalities and Related Topics, vol. 116 of North-Holland Mathematics Studies, North-Holland Publishing, Amsterdam, The Netherlands, 1985.

[13] F. John and L. Nirenberg, "On functions of bounded mean oscillation," Communications on Pure and Applied Mathematics, vol. 14, pp. 415-426, 1961.

[14] B. Muckenhoupt and R. L. Wheeden, "Weighted bounded mean oscillation and the Hilbert transform," Studia Mathematica, vol. 54 , no. 3, pp. 221-237, 1975/76. 


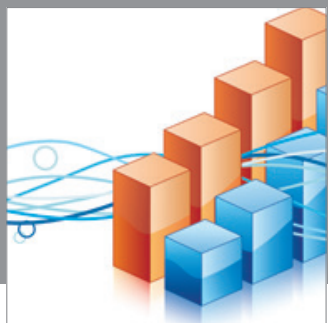

Advances in

Operations Research

mansans

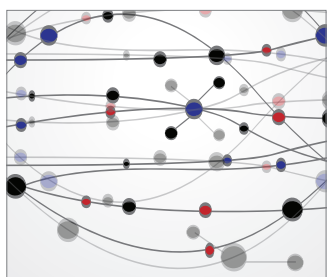

The Scientific World Journal
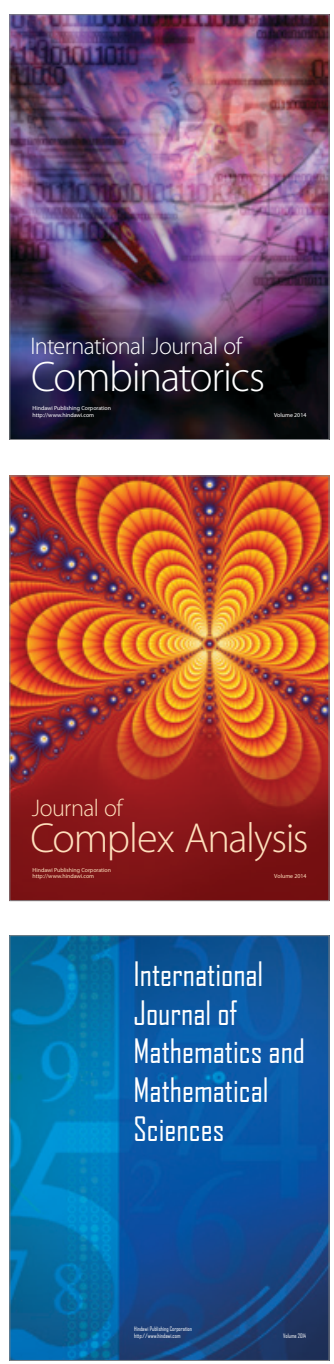
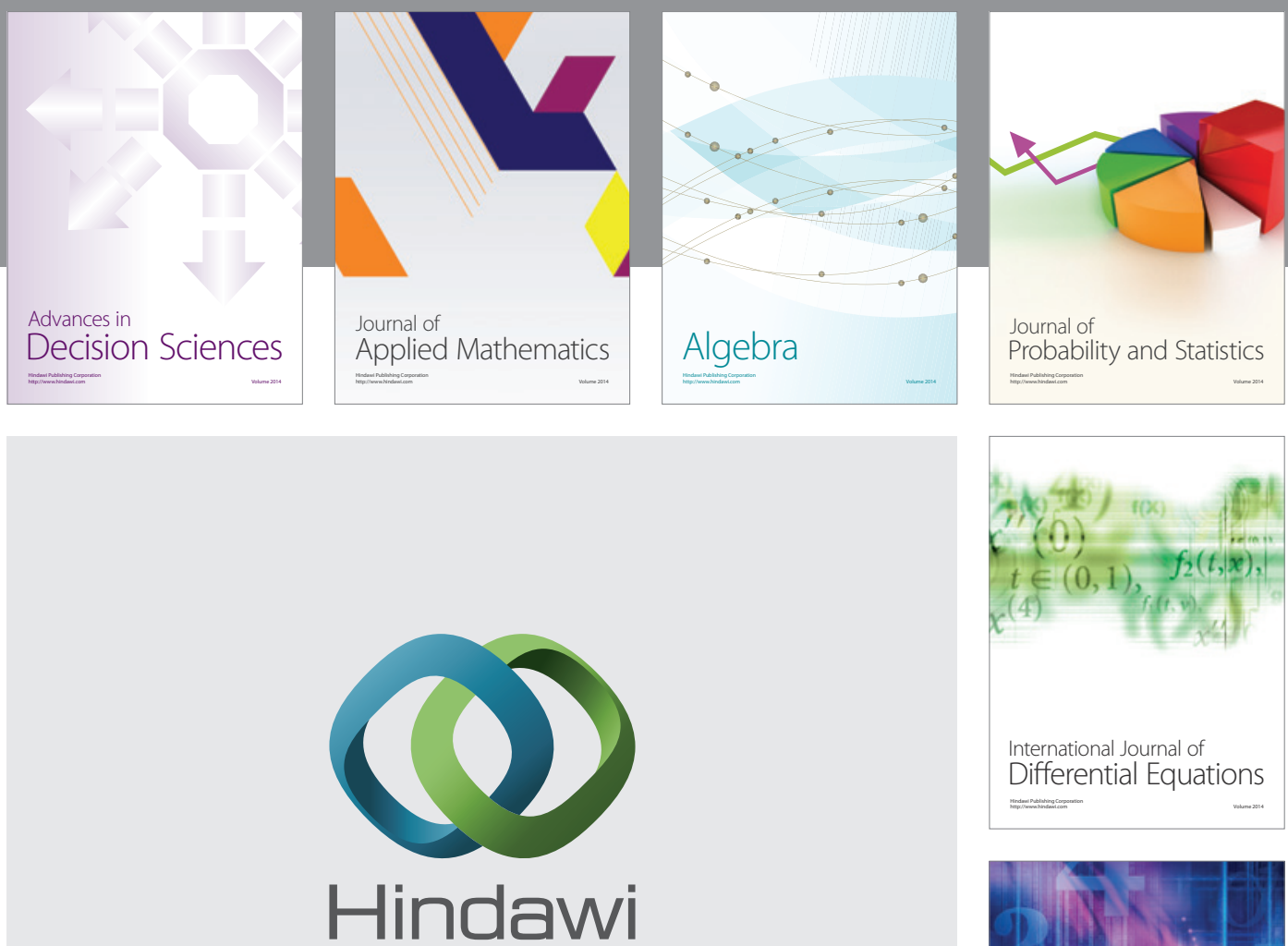

Submit your manuscripts at http://www.hindawi.com
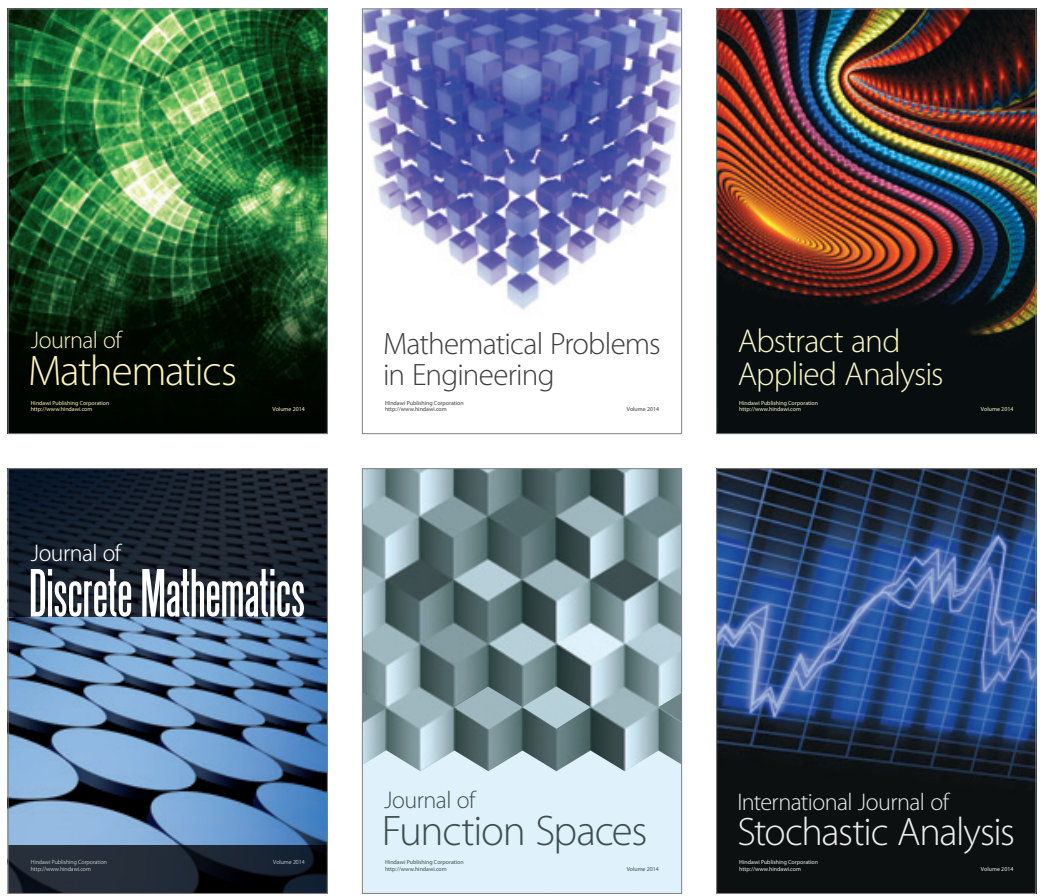

Journal of

Function Spaces

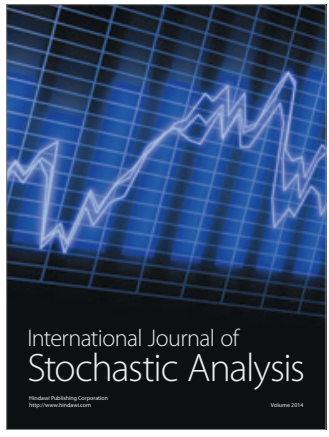

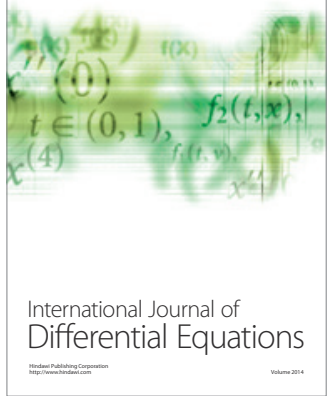
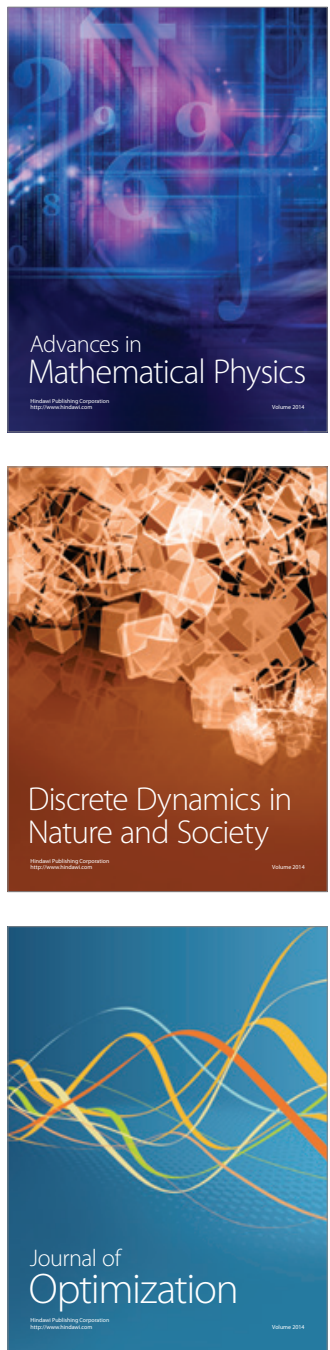